\title{
Super Resolution of MR Brain Images Using Compressive Sensing and Fuzzy Logical Rules
}

\author{
Charles Stud Angalakurthi ${ }^{1^{*}}$ \\ https://orcid.org/0000-0002-9069-520X
}

\section{Ramamurthy Nallagarla ${ }^{2}$ \\ https://orcid.org/0000-0002-6691-1718}

\begin{abstract}
1Jawaharlal Nehru Technological University Anantapur, Research Scholar, Department of ECE, Ananthapuramu, Andhra Pradesh, India; ${ }^{2}$ G.Pullaiah College of Engineering \& Technology, Professor in ECE Department, Kurnool, Andhra Pradesh, India.
\end{abstract}

Editor-in-Chief: Alexandre Rasi Aoki Associate Editor: Paulo Vitor Farago

Received: 2020.04.12; Accepted: 2020.10.22.

*Correspondence: mightystud727@gmail.com; Tel.: +91-9948942244 (C.S.A.)

\section{HIGHLIGHTS}

- In medical surround, high resolution images are needed to discern the details of image.

- Super resolution image can be made from one/few images by means of compressive sensing.

- A reckoning soft computing way for resolution enhancement, fuzzy logic is implemented.

- Better results are obtained for a dataset with different performance metrics.

Abstract: The proficiency of image processing is of extreme importance in perceiving and collecting information from the images, which includes the process of changing or interpreting existing images. In medical image processing, imaging with more accuracy plays a crucial role in better diagnosis or for the posterior analysis of treatment. Magnetic Resonance Imaging (MRI) is a medicinal creative tool for studying the internal structures and functionalities of human brain, knee, heart, liver, etc. Typical MR scans are essential now for better diagnosis but, limited resolution that is often inadequate for extracting detailed and reliable information. So, for the super resolution (SR) of MR brain images concepts of compressive sensing (CS) \& fuzzy logical rules to improve data quality are proposed in this paper. Usually, reconstruction of an SR image is the formation of high resolution $(\mathrm{HR})$ image which is obtained from one or few low resolution (LR) images. In the proposed method, with the help of compressive sensing a very limited number of images are considered even though it's a challenging task and fuzzy logical rules for a specific membership function are applied to improve the resolution of the image. To assess the performance of the proposal, different metrics are evaluated and achieved better results.

Keywords: low resolution; super resolution; compressive sensing; fuzzy logical rules. 
Angalakurthi, C.S. and Nallagarla, R.

\section{INTRODUCTION}

Medical imaging is a powerful structural characterising method which creates a sense modality for the human body interior parts for medial analysis and proceedings. It mainly processes lost, distorted, conflicting, redundant and vague information [1]. As brain is a crucial and analyzable organ, magnetic resonance imaging is the widely used mode for identification of brain abnormal conditions [2, 3].

Conventionally, radiologists analyzed MR images manually to detect the anomalous conditions of the brain. But it is very difficult and time taking to manual interpretation and intervention of the large number of images [4] which causes interobserver variability. Especially for the analysis of MR brain images, it is very essential to maintain the images with good resolution for the extraction of the clear and complete information quantitatively [5] for the posterior analysis. Generally, a high resolution image can be constructed from the more number of collected LR images and super resolution image is constructed from few number of LR images. So, to take the limited few samples an approach compressive sensing is used along with fuzzy morphology to improve the resolution of the image.

As MR brain images have low contrast histogram equalization can be used to enhance the quality of the images [6]. So, to improve the resolution of the image histogram processing is implemented. In basic histogram process the intensities are adjusted along the pixels which are taken at the probabilistic positions. In histogram with the help of cumulative distributive function (cdf) the pixel intensities are equalized [7]. As the basic histogram equalization process is a discriminative method, it may improve the contrast of the background noise in result false contours may appear in the image and it is also not applicable for smaller bright or dark regions. So, next to concentrate on the smaller regions with their local cdf's, an occurent of histogram called adaptive method used for better results $[8,9]$. But the problem in adaptive histogram processing is its inclination to over amplify noise in comparatively consistent regions of an image. So, to take over all the artefacts of the aforementioned methods here a fuzzy based approach is implemented.

As Taranbir Kaur and Ravneet Kaur Sidhu demonstrated in 2016 [10], fuzzy logic can be used for enhancement with the help of a specified membership function. Mainly fuzzy logic is used to handle the images which are having vagueness and uncertainty. The objective determination of quality of images can be done by mathematical functions, so fuzzy logic is suitable for quality measurement objectively [11].

P. Burillo and coauthors [12] demonstrated that basic fuzzy morphological operators either dilation or erosion can be used in the image processing and in 2017 Rajesh Muthu and coauthors [13] stated that with the help of a structuring element and morphological operators medical images quality can be improved. So, in the proposal fuzzy logical rules with a specified membership along with morphological operations are carried out based on fitting a structuring element to improve the resolution of the MR brain images.

In the area of MR imaging research, there is a remarkable development in deep learning to handle large image data management for quality improvement. So, deep learning is also having an underway to handle uncertain data of MR images. Therefore there can be the potential to use machine learning and/or deep learning for the posterior analysis of the MR brain images [14].

\section{MATERIAL AND METHODS}

In order to improve the resolution of the MR brain images in a better way is proposed when compared with aforementioned methods like twin concepts of compressive sensing and Fuzzy Logic are implemented. Compressive sensing which deals with the consideration of the single or few LR images for the reconstruction process. But as a challenging task, here only one LR sample will be taken for which the resolution will be improved. Compressive sensing is a new path in medical image processing which provides speed and quality of diagnosis. Especially in health monitoring, compressive sensing tackles high data volume of images having uncertainty and vagueness. With compressive sensing, instead of considering multiple LR images, only single image will be taken to make the accurate recovery.

Along with Compressed sensing, Fuzzy logical rules are applied which makes a pliable numerical structure for image processing. Fuzzy logic is an intelligent disguise probabilistic theory which allows one to specify appropriately and handle imperfect and uncertain data. Fuzzy logic doesn't need any model to handle imperfect data. Fuzzy image processing is a process to interpret the knowledge of human reasoning into intuitive tool to handle imperfect data. Fuzzy Environment comprises of the elements corresponds to membership and 
uncertainty functions which are associated with image properties. In the medical image processing an expert knowledge is usually exploited to represent the uncertain data which can be described as fuzzy If-then logical rules [10]. A classical rule uses only logical symbols 1 and 0 , whereas fuzzy rule consider a fuzzy set which represents the different levels of differentiation like low, medium and high values. The modelling of the fuzzy framework will be designed by rendering the underlying locution and uncertainty, carried by the image itself.

A morphological operation simplifies the images by protecting the important characteristics of the images. Morphological operations measures useful features by utilizing peculiar sequences of neighborhood transformations. Fuzzy morphology is a mathematical hypothetical process used to minimize the vagueness and uncertainty in the image information. Fuzzy morphological operations either dilation or erosion can be used to improve the resolution with the selection of proper structuring element.

\section{Image Resolution Enhancement Process Flow}

The process flow sequence of the proposed fuzzy method for the resolution enhancement is shown in Figure 1. As shown in Figure 1, the reference data set is scaled down for creating LR images and it is filtered to remove any type of noise. With the means of compressive sensing for an LR image fuzzy morphological operations are applied for super resolution of the image. And there can be the potential to upload the images to Machine learning/ Deep learning for the posterior analysis/ further processing.

A fuzzy logical rule can be of the form as a counterfactual statement which is characterized with linguistic variables and linguistic values. Usually it can be defined with antecedent and consequent operations as [15].

IF $x$ is $A$ then $y$ is $B$, Where $x$ and $y$ are linguistic variables and $A, B$ are linguistic values.

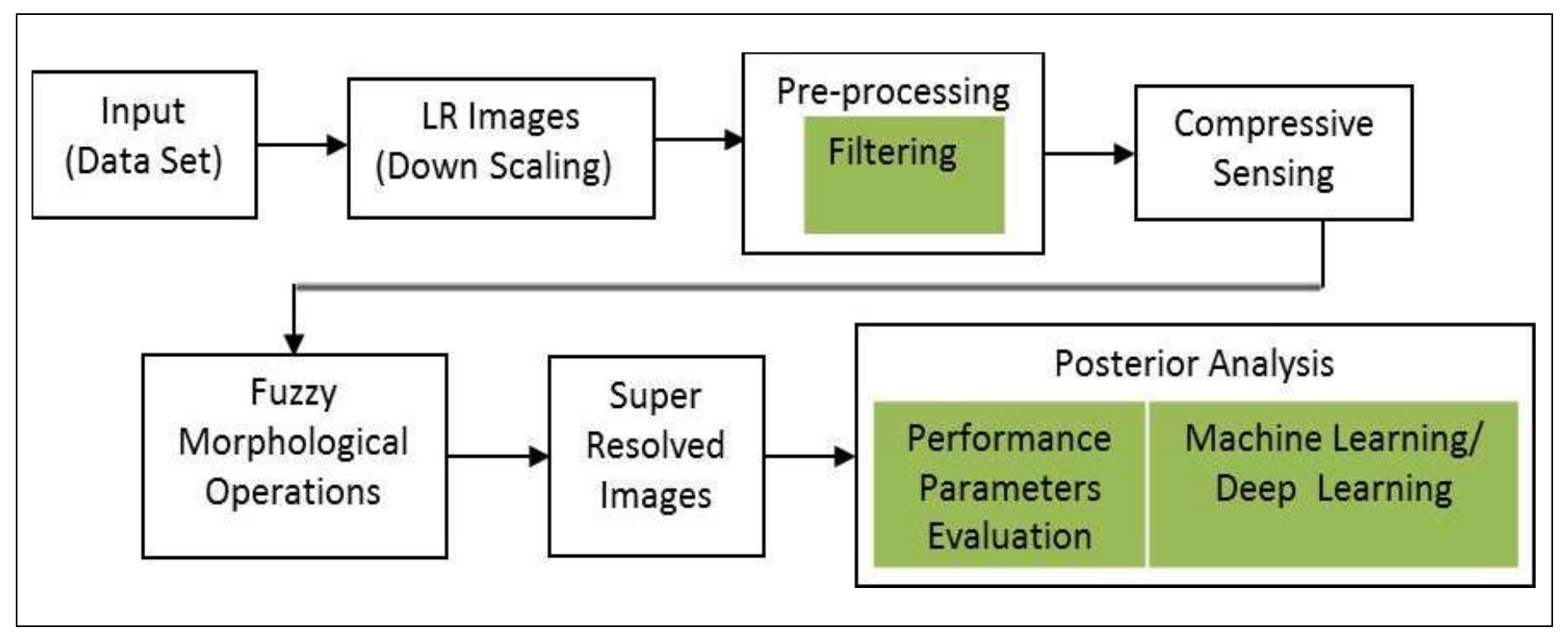

Figure 1. Process flow for the proposed method

In the proposal, for the resolution enhancement of the MR brain image, for an image a fuzzy set will be formed which categorizing the intensity values as low, medium and high ranges. To make an SR image the lower and mid-range intensities are scaled up and the complement of the image will be taken with a specified membership function as shown in the equations (1) and (2) respectively. The scaled up intensities over an image will be formed as a fuzzy subset [16].

$$
\bar{A}=X-A,
$$

Where $X$ is the LR image, $A$ is the fuzzy subset formed with scaling over $X$ and $A$ is the complement of $A$. And the membership function of the complement $A$ is defined as [17]

$$
\mu_{\bar{A}}(x)=1-\mu_{A}(x)
$$


With the help of the formation of a specified fuzzy set, a morphological operation will be implemented to improve the resolution of the image. Here, dilation morphological operation [18] is applied to the fuzzy membership function so that the resolution increases with the full structuring element [19]. And the proposed algorithm is summarized as follows:

\section{Algorithm:}

Step1: Take a reference image

Step2: Scaling to get an LR image

Step3: Fuzzy antecedent and consequent operations

Step4: Membership function generation

Step5: Fuzzy dilation.

Fuzzy morphology is applied to the taken data set of six images along with conventional methods i.e. histogram and adaptive histogram processing techniques and are shown in the Figure 2(a)-(e).

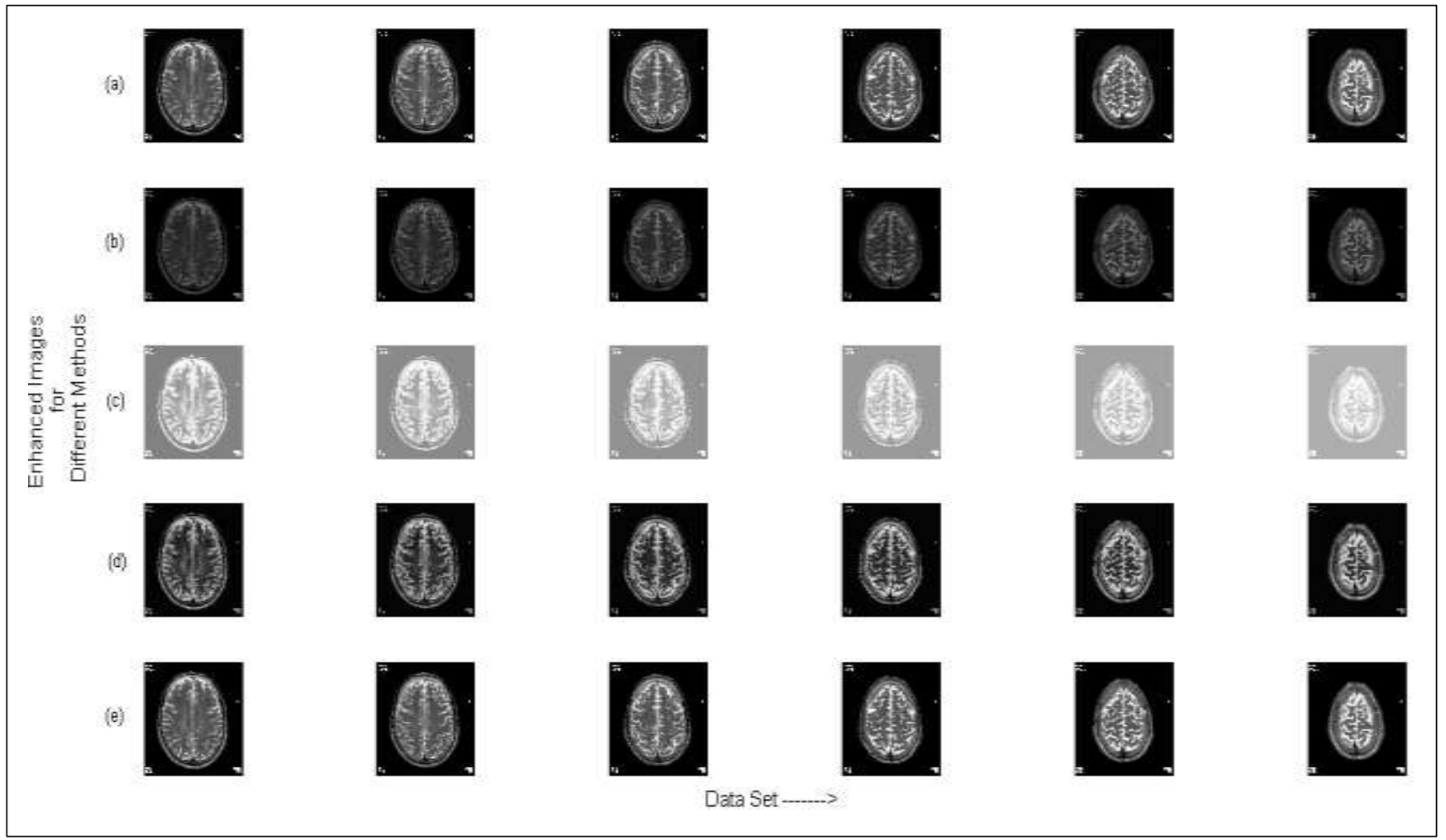

Figure 2. Enhancement of Images for different methods of a data set of six images (a)-(e). (a) Reference Data Set; (b) LR images; (c) Histogramed Images; (d) Adaptive histogramed images; (e) Images based on fuzzy morphology.

In Figure 2(a), it shows the reference data set, 2(b) represents the scaled version of images which are LR images, 2(c) and 2(d) shows the histogramed and adaptive histogramed images and 2(e) shows the fuzzy morphological images for the specific membership function respectively. 


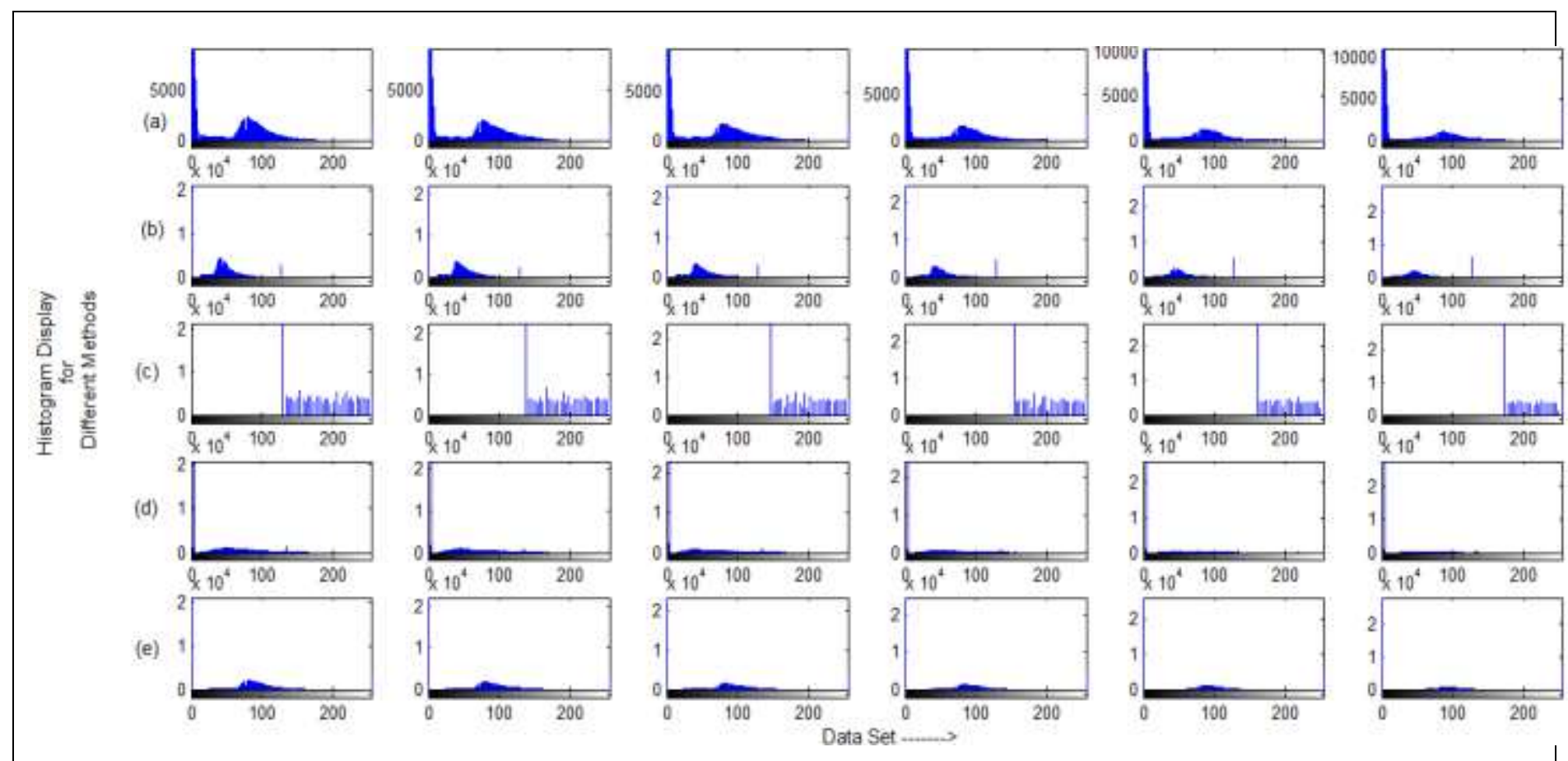

Figure 3. Histogram representation of the data set (a)-(e) for different methods(a)Reference data set; (b) LR images; (c) histogram processing; (d) adaptive histogram; (e) histogram for fuzzy morphology.

As well as for the data set, histogram equalization is implemented and their histogram representations are shown in Figure 3(a)-(e) respectively. And it is observed that the peak of the histogram is much better for fuzzy approach than the histogram and adaptive methods.

\section{Image Performance Metrics}

Usually visual comparison is not adequate for the quantitative assessment of the any technique. So, different performance metrics are to be evaluated to assess quantitatively. In the proposal three parameters are assessed i.e. peak signal to noise ratio (PSNR), absolute mean brightness error (AMBE) and entropy (E).

PSNR is a degree of enhancement used to estimate achievement attribute between the original and transformed images $[20,10]$. A high PSNR represents that quality is better than the input image.

$$
P S N R=20 \log _{10}\left(\frac{\text { MAX }_{f}}{\sqrt{M S E}}\right),(\ln \mathrm{dB})
$$

Where MAXf is the highest signal power in the original image and MSE is the accumulative squared error between the original and reconstructed images.

AMBE represents the mean brightness preservation which exhibits the difference between mean brightness of input and output images [21].

$$
\mathrm{AMBE}=|\mathrm{m}(\mathrm{x})-\mathrm{m}(\mathrm{y})| \text {, }
$$

Where $\mathrm{m}(\mathrm{x})$ and $\mathrm{m}(\mathrm{y})$ are the mean brightness of the input and output images respectively.

Another important parameter which represents the quantitative treatment for recognizing the details contained in an image i.e. entropy. Entropy is a measure of fuzziness and it can be defined as harmonizing state of the intensity level with reference to the pixel value [22]. Usually representation for entropy, $E$ is given by

$$
\left.E=-\sum_{i=1}^{L} m_{i} \log _{2} m_{i}\right) \text {, }
$$

Where $i$ represent the greylevel, $\mathrm{m}$ is the probable existence of greylevel of $i$ and $L$ is the total number of greylevels. 


\section{RESULTS AND DISCUSSION}

PSNR, AMBE and entropy values are evaluated for the fuzzy and conventional approaches and the comparison is tabulated below in tables 1, 2 and 3 for a data set of six images (image 1-image 6). With the concept of compressive sensing, instead of taking multiple images only one image is taken from the data set at a time.

From table 1, it can be determined that the PSNR value for the proposed fuzzy technique is improved than the conventional methods. As the PSNR is better for the fuzzy, the visuality of the image resolution will be much better so that the perception of the details will be increased. As the resolution is improved, discern details of the MR brain image can be accurately collected for the critical instances.

Table 1. PSNR values for the three approaches

\begin{tabular}{lcccccc}
\hline Method & Image1 & Image2 & Image3 & Image4 & Image5 & Image6 \\
\hline Histogram & 6.93 & 6.55 & 6.14 & 5.63 & 5.12 & 4.40 \\
Adaptive & 23.00 & 22.42 & 22.03 & 22.53 & 23.31 & 23.90 \\
Fuzzy & 24.21 & 23.73 & 23.33 & 23.34 & 23.79 & 24.31 \\
\hline
\end{tabular}

From table 2, it can be seen that that brightness error is reduced to so much extent (almost negligible) with fuzzy approach and is easy to differentiate the reconstructed image with original image.

Table 2. AMBE values for the three approaches

\begin{tabular}{lcccccc}
\hline Method & Image1 & Image2 & Image3 & Image4 & Image5 & Image6 \\
\hline Histogram & 113.20 & 117.91 & 122.92 & 129.98 & 137.56 & 149.29 \\
Adaptive & 3.33 & 3.76 & 4.16 & 3.50 & 2.76 & 2.45 \\
Fuzzy & 2.48 & 1.50 & 0.79 & 0.75 & 0.95 & 0.70 \\
\hline
\end{tabular}

From table 3, it can be found that entropy for the data set is improved with fuzzy approach i.e. average information will be more which helps to take decisions in critical instances. Entropy measure makes it easy to reconstruct the original information from the LR images. Improving the entropy gives determining the circumstantial information regarding the images.

Table 3. Entropy Values for the three approaches.

\begin{tabular}{lcccccc}
\hline Method & Image1 & Image2 & Image3 & Image4 & Image5 & Image6 \\
\hline Histogram & 3.39 & 3.18 & 2.97 & 2.76 & 2.56 & 2.24 \\
Adaptive & 4.66 & 4.54 & 4.37 & 4.08 & 3.77 & 3.39 \\
Fuzzy & 4.76 & 4.63 & 4.46 & 4.16 & 3.81 & 3.42 \\
\hline
\end{tabular}

For the analysis, graphical representation of the fuzzy approach with the conventional methods is shown in Figure 4(a), (b) and (c). And it can be noticed that better values are obtained when assimilated with conventional methods. 


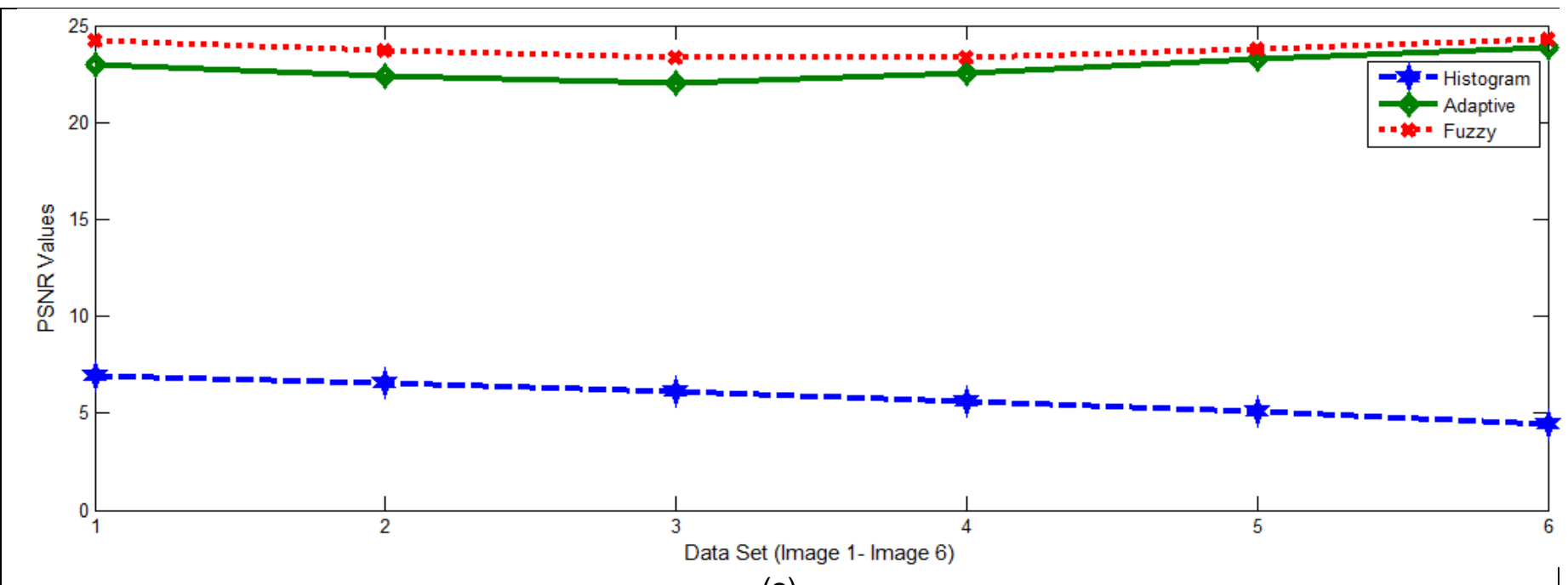

(a)

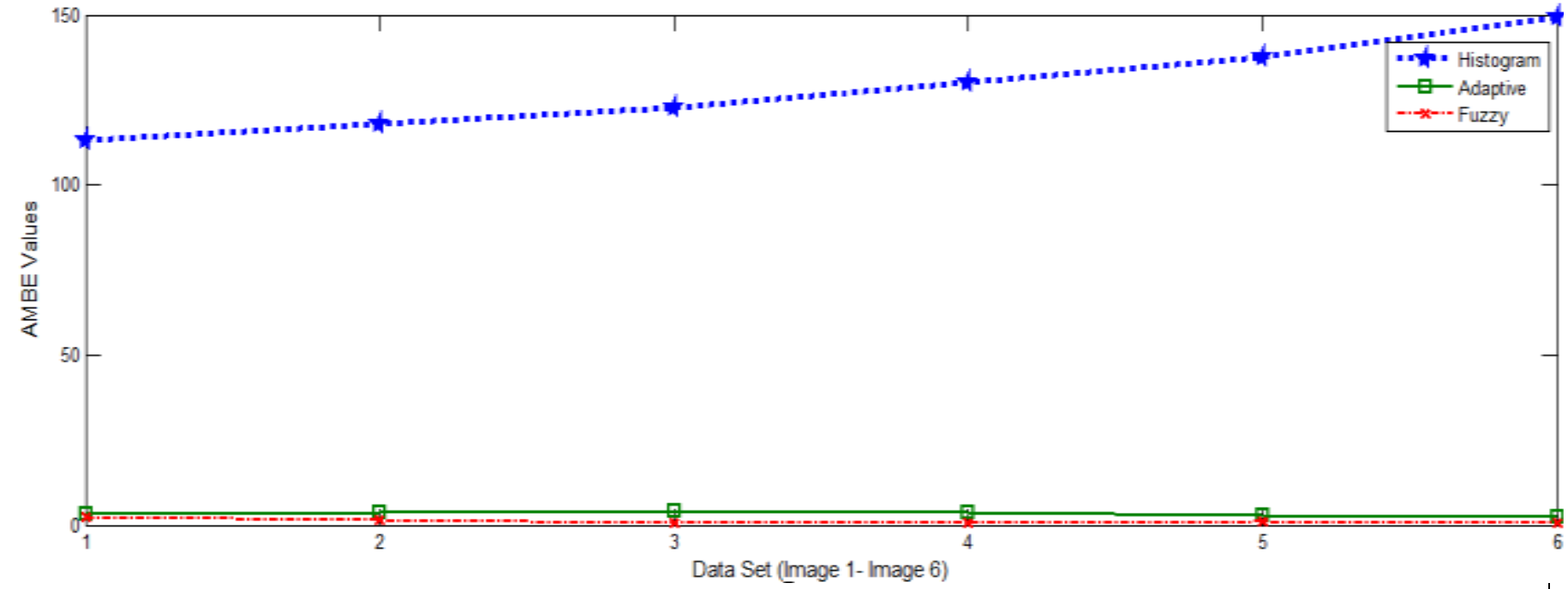

(b)

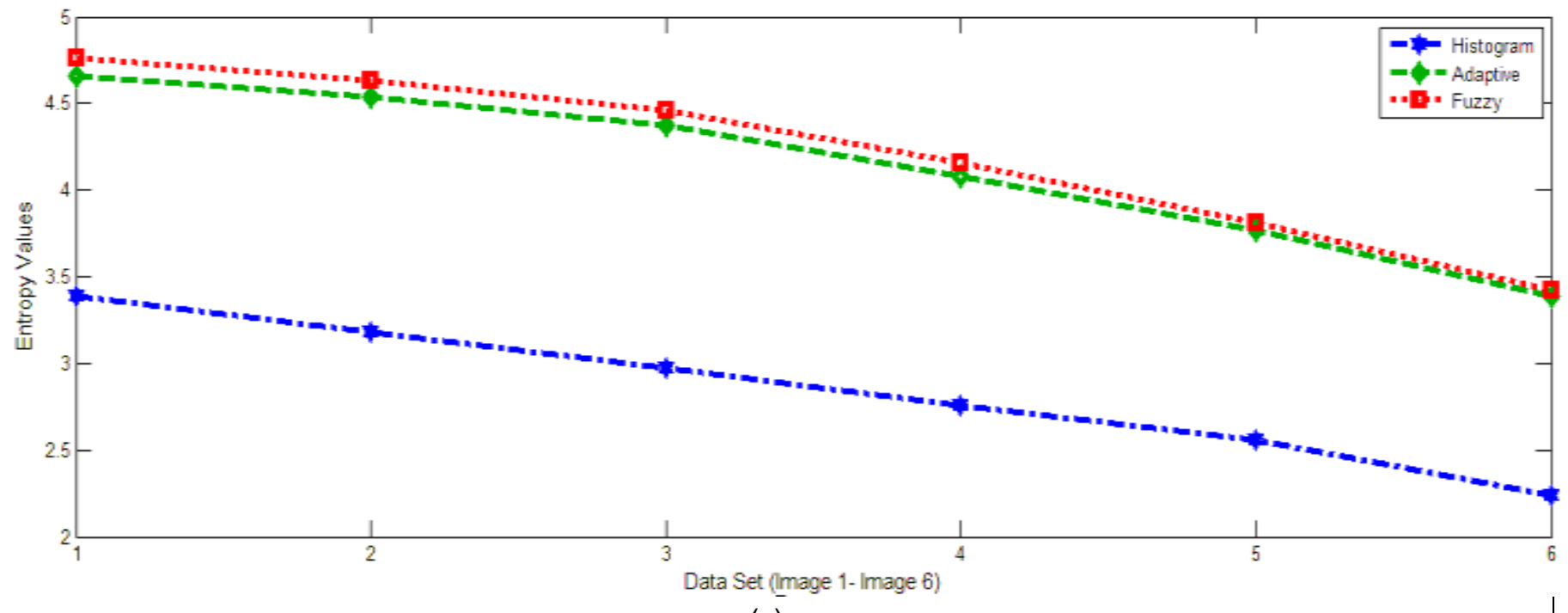

(c)

Figure 4. Graphical representation of three perforemance metrics. (a)PSNR values comparison; (b) AMBE values comparison; (c) Entropy values comparison. 
Angalakurthi, C.S. and Nallagarla, R.

In Figure 4(a), image metric PSNR is analyzed graphically and it is observed that PSNR is improved with the fuzzy morphology. As PSNR is high, easy to understand ang gather the complete disernible details from the reconstructed MR brain images. The brightness error measurement AMBE is visualized in Figure 4(b), in which the proposed fuzzy technique reduced the error to very negligible value between the input and output images. $A$ very low value of $A M B E$ represents the high quality image reconstruction. The parameter which represents the image information content, entropy measurement is analyzed in Figure 4(c). Increase in the entropy value interprets that the average uncertainity information.

\section{CONCLUSION}

In this paper fuzzy mathematical morphology is developed for the MR brain image resolution enhancement. The superior performance of the proposed approach is because of fuzziness i.e. formation of the fuzzy set with antecedent and consequent operations for a specific membership function. This Fuzzification improves effectively the PSNR, Entropy and reduces the error with less computation time compared with the conventional methods. As the quality or visuality of the image is increased, perception of the details from the image will be easy and moreover reliable information can be collected which helps in better diagnosis/treatment. In the future, there can be the potential to upload the super resolved images to cloud for machine learning/ deep learning for further analysis for the better treatment/diagnosis to save the mankind.

\section{REFERENCES}

1. Khan A, Li JP, Shaikh RA. Medical image processing using fuzzy logic. In2015 12th International Computer Conference on Wavelet Active Media Technology and Information Processing (ICCWAMTIP) 2015 Dec 18 (pp. 163-167). IEEE.

2. Raghavendra U, Tan R, Edward JC, Rajendra AU, Anjan G. Application of multiresolution analysis for automated detection of brain abnormality using MR images: A comparative study. Future Generation Comp Syst. 2019 January; 90: 359-67.

3. Ribbens A, Hermans J, Maes F, Vandermeulen D, Suetens P. Unsupervised segmentation, clustering, and groupwise registration of heterogeneous populations of brain MR images. IEEE transactions on medical imaging. 2013 Jun 19; 33(2):201-24.

4. Talo M, Baloglu UB, Yıldırım Ö, Acharya UR. Application of deep transfer learning for automated brain abnormality classification using MR images. Cogn. Syst. Res. 2019 May 1; 54:176-88.

5. Moeskops P, Viergever MA, Mendrik AM, De Vries LS, Benders MJ, Išgum I. Automatic segmentation of MR brain images with a convolutional neural network. IEEE transactions on medical imaging. 2016 Mar 30; 35(5):1252-61.

6. Wang X, An Z, Wang H, Chang Y. MR brain image enhancement via learning ensemble. In2018 IEEE International Conference on Intelligence and Safety for Robotics (ISR) 2018 Aug 24 (pp. 282-285). IEEE.

7. Lavania KK, Kumar R. A comparative study of image enhancement using histogram approach. Int. J. Comput. Appl. 2011; 32(5):1-6.

8. Kaushik P, Gupta U. A Modified BPDHE Enhancement Algorithm for Low Resolution Images. In Proceedings of Sixth International Conference on Soft Computing for Problem Solving 2017 (pp. 10-20). Springer, Singapore.

9. Lu L, Zhou Y, Panetta K, Agaian S. Comparative study of histogram equalization algorithms for image enhancement. In Mobile Multimedia/Image Processing, Security, and Applications 20102010 Apr 28 (Vol. 7708, p. 770811). International Society for Optics and Photonics.

10. Kaur T, Sidhu RK. Optimized adaptive fuzzy based image enhancement techniques. International Journal of Signal Processing, Image Processing and Pattern Recognition. 2016; 9(1):11-20.

11. Mittal P, Saini RK, Jain NK. Image Enhancement Using Fuzzy Logic Techniques. Soft Computing: Theories and Applications; Proceedings in Advances in Intelligent Syst and Computing. 2019; 742: 537-45.

12. Burillo P, Frago N, Fuentes R. Fuzzy morphological operators in image processing. Mathware and Soft Computing. 2003; 10(2/3):85-100.

13. Rajesh M, Saritha S, Rani C, Pearl Mary S. Morphological Operations in Medical Image Pre-Processing. IEEE International Conference on Advanced Computing and Communication Systems; 2017; p. 2065-70.

14. Lee D, Lee J, Ko J, Yoon J, Ryu K, Nam Y. Deep learning in MR image processing. Investigative Magnetic Resonance Imaging. 2019; 23(2):81-99. 
15. Souverville S, Rosales JA, Funes FJ, Dehesa M, Hernández IV, Lozano LV. Fuzzy Logic Applied to Improvement of Image Resolution using Gaussian Membership Functions. Res. Comput. Sci.. 2015 Dec; 102:77-88.

16. Solovyov SA. Fuzzy algebra as a framework for fuzzy topology. Fuzzy Sets and Systems. 2011 June; 173 (1): $81-99$.

17. Chaira T. Medical image enhancement using intuitionistic fuzzy set. In2012 1st International Conference on Recent Advances in Information Technology (RAIT) 2012 Mar 15 (pp. 54-57). IEEE.

18. Oh J, Hwang $\mathrm{H}$. Feature enhancement of medical images using morphology-based homomorphic filter and differential evolution algorithm. Int J Control Autom Syst. 2010 Aug 1; 8(4):857-61.

19. Baets BD, Kerre E. The Fundamentals of Fuzzy Mathematical Morphology. Int J of General Syst. 2015; 23: 155-71.

20. Singh LS, Ahlawat AK, Singh KM, Singh TR, Singh YS. Medical Image Enhancement using Fuzzy and Regression based Neural Network Approach. Int. J. Appl. Eng. Res. 2019; 14(7):1532-8.

21. Chang $\mathrm{Y}$, Jung $\mathrm{C}, \mathrm{Ke} \mathrm{P}$, Song $\mathrm{H}$, Hwang J. Automatic contrast-limited adaptive histogram equalization with dual gamma correction. IEEE Access. 2018 Jan 25; 6:11782-92.

22. Tsai DY, Lee Y, Matsuyama E. Information entropy measure for evaluation of image quality. J Digit. Imaging. 2008 Sep; 21(3):338-47.

(c) 2021 by the authors. Submitted for possible open access publication under the terms and conditions of the Creative Commons Attribution (CC BY NC) license (https://creativecommons.org/licenses/by-nc/4.0/). 